\title{
sciendo
}

\author{
BULGARIAN ACADEMY OF SCIENCES
}

CYBERNETICS AND INFORMATION TECHNOLOGIES • Volume 19, No 3

Sofia • $2019 \quad$ Print ISSN: 1311-9702; Online ISSN: 1314-4081

DOI: $10.2478 /$ cait-2019-0029

\section{Mitigating Hotspot Issue in WSN Using Sensor Nodes with Varying Initial Energy Levels and Quantification Algorithm}

\author{
R. Balamurali ${ }^{1}$, K. Kathiravan ${ }^{2}$, T. Krishnan ${ }^{1}$ \\ ${ }^{1}$ Department of CSE, The ICFAI Foundation for Higher Education, IcfaiTech, Hyderabad, India \\ ${ }^{2}$ Department of IT, Easwari Engineering College, Ramapuram, Chennai, India \\ E-mails: mohmur@gmail.com kathirraji@gmail.com tkrishnan@ifheindia.org
}

\begin{abstract}
A typical Wireless Sensor Network (WSN) uses multihop communication rather than direct transmission. In a multi-hop communication, the sensor node communicates the sensed data to its neighbor node, which is comparatively closer to the sink and the receiving node will forward the data to its neighbor node. This process continues until the data reaches the sink. Due to the multihop communication, the nodes closer to the sink have to transmit and receive more data and control packets compared to other nodes. Hence, the nodes closer to sink may deplete their energy at a faster rate and may die soon. This may create network isolation. This issue is called as the Hotspot problem. In this paper, we are proposing a Quantification algorithm for Sensor Nodes with varying Initial Energy Level to mitigate the Hotspot effect.
\end{abstract}

Keywords: WSN, hotspot, multihop, tier, SMAC (Sensor MAC), RIMRP (Refined Integrated MAC and Routing Protocol).

\section{Introduction}

A Wireless Sensor Network (WSN) is a set of sensor nodes deployed in a given area to sense environmental parameters like heat, pressure, temperature, light intensity etc. These sensor nodes can auto configure to form a WSN (Fig. 1). Each sensor node is equipped with limited Computing, Communicating and Sensing capability. Most commonly, WSNs are used for remote surveillance; typical examples are Forest Fire Surveillance, Battlefield Surveillance, and Structural Health Monitoring etc. The sensor nodes are battery powered. Hence, energy is a scarce resource in WSN and efficient energy utilization is one of the core research objectives of WSN. The hotspot is an issue related to uneven or inefficient energy consumption. Based on the type of sensor nodes used, a WSN can be classified as Homogeneous WSN or Heterogeneous WSN. Sensor nodes are likely to have identical sensing, computing, communication and energy capabilities in a Homogeneous WSN. The sensor nodes have varying 
sensing, computing, communication and energy capabilities in a Heterogeneous WSN (Fig. 2).

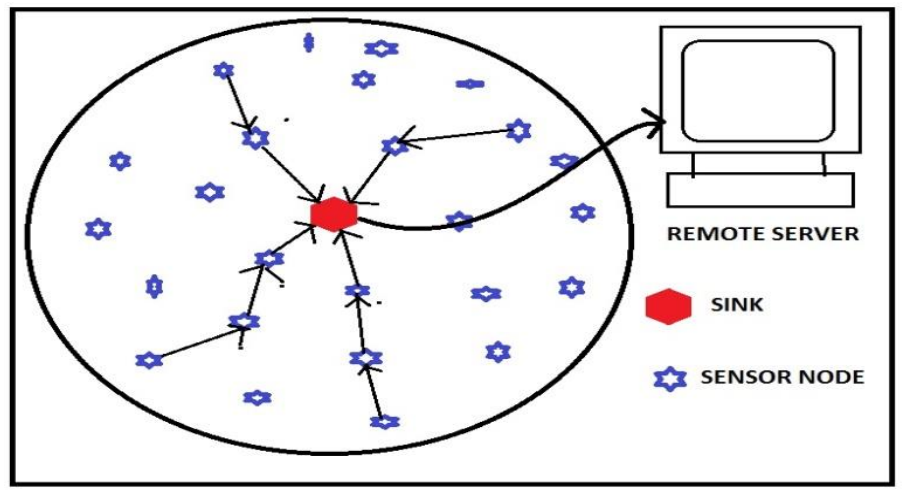

Fig. 1. A typical WSN

Due to the advancement in MEMS (Micro-Electro-Mechanical Systems) the availability of low cost sensor nodes with varying battery levels has drastically increased. This leads to a lot of research in WSN. Hotspot problem is an issue related to inefficient energy distribution. Due to the inherent nature of multihop (hop-to-hop) communication in WSN, the nodes closer to the sink has to send and receive more data and control packets compared to other nodes. Hence, they may deplete their energy at a faster rate and may result in network isolation.

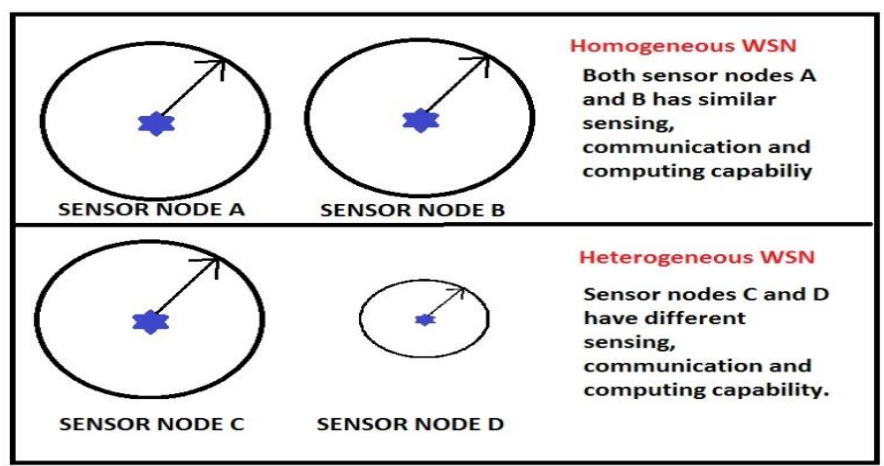

Fig. 2. Homogeneous WSN vs. heterogeneous WSN

Several approaches are discussed in literature to mitigate the Hotspot effect. This can be broadly classified into four categories viz., Transmission Range Optimization, Sensor Deployment Strategy, Adding more nodes around the sink, and using Sensor Nodes with varying initial energy (battery) levels.

Transmission Range Optimization: Different Sensor nodes use different transmission range based on its distance from the sink, i.e., the nodes closer to the sink will have a shorter transmission range and the nodes away from sink will have a longer transmission range. Using such intelligent power control technique the impact of Hotspot can be reduced to a certain extent. 
Sensor Deployment Strategy: The most commonly used sensor or node deployment strategy to mitigate Hotspot effect is Movement of Data Sink: When Hotspot occurs, the sink node will be relocated to some other location, so as to reduce or to mitigate the impact of Hotspot. Deployment of Multiple Base stations: Rather than using single sink or base station, this technique uses multiple base stations to reduce the impact of the Hotspot.

Adding More Nodes around Sink: A simple and efficient technique to mitigate Hotspot problem in Wireless Sensor Network is to place additional sensor nodes around Sink. Adding more nodes around sink will balance energy distribution and will reduce the impact of the Hotspot.

Sensor Nodes with varying Initial Energy (Battery) levels: This technique uses sensor nodes with varying battery level, i.e., the nodes closer to the sink will be equipped with high energy level batteries and the nodes away from sink will have batteries of low energy levels. This technique uses nodes with varying energy levels to balance energy consumption and thereby reduces the impact of Hotspot.

\subsection{Protocol stack of WSN}

The protocol stack of WSN comprises [1] the Physical layer, Data link layer, Network layer, Transport layer, Application layer and it also consists of Power Management Plane, Mobility Management Plane, and Task Management Plane (Fig. 3).

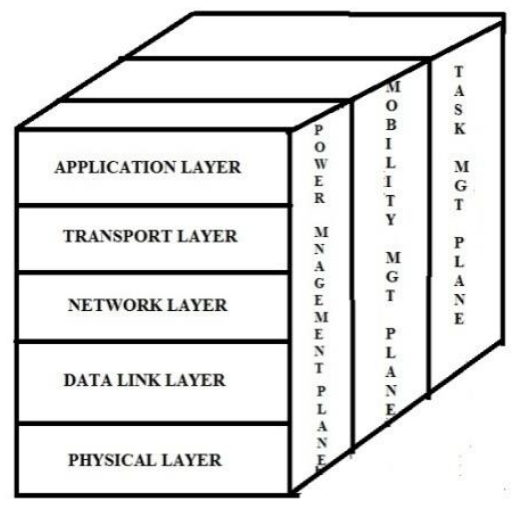

Fig. 3. WSN protocol stack

Physical Layer: It tackles the requirements of modulation, transmission and receiving methods used.

Data link Layer: It is accountable to ensure reliable communication through error control techniques and handle channel access through the MAC (Media Access Control) to decrease collision with nearby nodes.

Network Layer: It takes care of routing the data packets through routing algorithms like DSR (Dynamic Source Routing), AODV (ad hoc On-demand Distance Vector), and AOMDV (Ad hoc on Demand Multiple path Distance Vector), etc... 
Transport Layer: It facilitates to preserve the flow of data if the sensor network application needs it.

Power Management Plane: It administers how a sensor node utilizes its power, e.g., when the power level of the sensor node is modest, the sensor node broadcasts to its nearby nodes that, "it is low in power and cannot participate in routing messages". The left over power is set aside for sensing.

Mobility Management Plane: It identifies and records the movement of the sensor nodes.

Task Management Plane: It balances and schedules the sensing tasks given to a specific region. Not all sensor nodes in that region are required to perform the sensing task at the same time.

\subsection{Applications of WSN}

WSN has a wide range of applications starting from battlefield surveillance to Home Monitoring. The typical applications of WSN [2] are Battlefield Surveillance, Forest Fire Surveillance, Water Quality Monitoring, Landslide Detection, and Structural Health Monitoring, etc.,

\subsection{Energy efficiency in WSN}

Energy is a scarce resource in WSN [3]. Mostly, WSN is used for remote surveillance where a large number of tiny sensor nodes are deployed. The batteries used by the sensors are not replaceable, as they are deployed in a remote location where human reachability is tedious. Hence, the sensor nodes should go into alternate sleep and wake-up mode to effectively utilize the energy. When a sensor node is in sleep mode, it switches-off its antenna and other electronics and consumes the least power as possible. Again, after a certain amount of time it will come back to wake-up mode. This mechanism helps to effectively utilize energy. Ineffective energy utilization may lead to the following issues [3].

- Network isolation: If energy is not evenly used by all sensor nodes, the network may get isolated, due to critically low energy levels in some nodes.

- Energy holes: If some node performs more transmission and reception than other nodes, then these nodes will die quickly. Hence, an energy hole may be created, i.e., these nodes cannot take part in routing the data packets.

- Decreased network lifetime: Network lifetime is defined as the time at which the first node dies. If energy efficient protocols are not used, then it will have an impact on the network lifetime.

- Uneven energy distribution: If the energy utilization is not evenly distributed, it will lead to uneven energy levels in different parts of the sensor network.

- Ineffective surveillance: Ultimate aim of using WSN is remote surveillance. Due to the inefficient energy utilization, the surveillance may be ineffective.

\subsection{Hotspot problem in WSN}

Hotspot problem is an issue related to inefficient energy consumption. This research primarily focuses on mitigating the hotspot effect in WSN. A Typical WSN uses 
multi-hop communication rather than direct communication, i.e., the sensor nodes communicate their sensed parameter to the sink in a hop-by-hop approach, instead of direct communication. Due to multi-hop communication, the sensor nodes closer to the sink have to transmit and receive more packets when compared with other nodes. Hence, the nodes closer to the sink will consume more energy when compared with other nodes and they tend to die early leading to network isolation. This issue is called as Hotspot problem (Fig. 4). The Hotspot problem comes under energy efficiency in Wireless Sensor Network. Many energy efficient protocols are proposed in the literature to reduce the overall energy consumption of a sensor network. But, there are not sufficient researches to mitigate the hotspot effect. This work focuses specifically on mitigating hotspot problem in WSN.

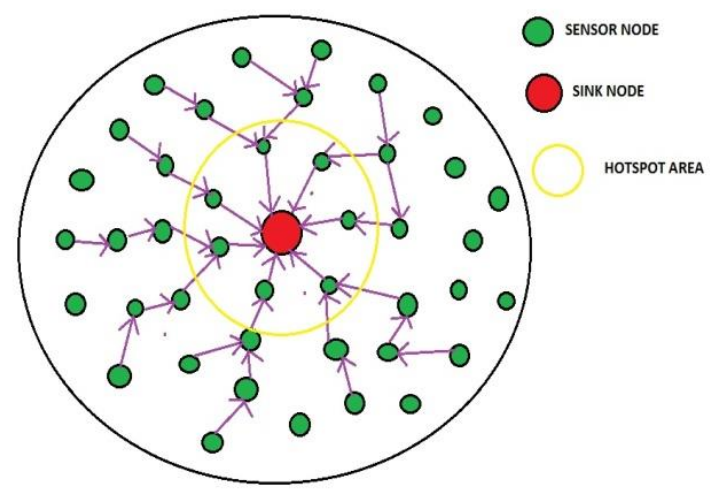

Fig. 4. Hotspot problem in WSN

\subsection{Research motivation}

A good amount of research is carried out on energy efficiency in Wireless Sensor Networks. The protocols LEACH [4], HEED [5], PEGASIS [6], Energy Efficient Unequal Clustering [7], Energy Efficient Clustering [8], and DEAR [9] are available in the literature. These protocols are designed with the intention to minimize the overall energy consumption of the sensor network. However, they fail to mitigate the Hotspot problem in WSN. There is not a good deal of research on mitigating the hotspot problem.

Some of the above-mentioned protocols [7,9] partly concentrate on mitigating the hotspot effect, but they are not much effective. AIMRP [10] introduces an integrated MAC and routing protocol. It divides the WSN into several tiers and packets are transferred from one tier to another by MAC control packets, thus it integrates the MAC and Routing protocol and avoids the overhead involved in routing. AIMRP is also designed with the intention to reduce overall energy consumption of the network and not to mitigate the hotspot effect.

Rivas, Voigt and Dunkels [11] provides a simple and efficient technique to mitigate the hotspot effect by adding additional sensor nodes around the sink. It was also proved analytically and through simulation. But they failed to provide an idea on how much sensors should be added and where should they be placed. 
Most of the above mentioned approaches try to reduce the overall energy consumption of the network and not specifically focus on mitigating the hotspot effect. Though some approaches $[9,11-13]$ try to mitigate the hotspot effect, they have not provided a clear idea about effective distribution of nodes/energy to increase the network lifetime and to reduce the impact of the hotspot. This scenario is the Motivation for this research. It aims at mitigating the Hotspot problem by using energy efficient Refined Integrated MAC and Routing Protocol (RIMRP). We also propose Quantification algorithm for sensor nodes with varying initial energy levels, which gives a direction about how the nodes with different energy levels should be distributed among the various tiers to reduce the impact of the Hotspot.

\subsection{Mathematical representation of the Hotspot problem}

Let the number of sensor nodes in a WSN be $N$.

Let the sensor network be divided into $n$ circular tiers, with sink in the middle.

Let the number of nodes in each tier be $x_{i}$ where $i$ represents the tier number.

The nodes are operating at a frequency of $2.4 \mathrm{GHz}$.

The algorithm for communication is based on refined AIMRP protocol which is explained below in the proposed work.

Let $T_{x}$ represent the energy required to transmit a packet.

Let $R_{x}$ represent the energy required to receive a packet. Then, the energy consumption in a tier can be represented as

$$
E_{i}=\frac{\left[N-\sum_{k=i+1}^{n} x_{k}\right]}{x_{i}} R_{x}+\frac{\left[N-\sum_{k=i}^{n} x_{k}\right]}{x_{i}} T_{x} .
$$

From that equation, it can be found that $\operatorname{MAX}\left\{E_{i}\right\}=E_{1}, E_{1}$ represents the energy consumed at Tier 1, which shows that Hotspot will occur at Tier 1.

\section{Related works}

In this section, we provide a brief introduction to various existing techniques to increase the network lifetime.

DEAR [9] optimized each individual distance so that all sensor nodes consume their energy at a similar rate. DEAR algorithm has a better performance in energy consumption as well as network lifetime.

The energy model used by DEAR is presented here. Each sensor node consumes $E_{T x}$ amount of energy to transmit $l$-bits message over distance $d$. This is given as

$$
E_{T_{x}}(l, d)=\left\{\begin{array}{c}
l \cdot E_{\text {elec }}+l \cdot \varepsilon_{\mathrm{fs}} \cdot d^{2} \text { if } d<d_{0}, \\
l \cdot E_{\text {elec }}+l \cdot \varepsilon_{\mathrm{amp}} \cdot d^{4} \text { if } d \geq d_{0} .
\end{array}\right.
$$

Each sensor node consumes $E_{R_{x}}$ amount of energy to receive this message. This is given as

$$
E_{R_{x}}(l)=l . E_{\text {elec }} .
$$

Each sensor node consumes $E_{F_{x}}$ amount of energy to forward this message. This is given as 


$$
E_{F_{x}}(l, d)=E_{T_{x}}(l, d)+E_{R_{x}}(l)=\left\{\begin{array}{c}
2 l \cdot E_{\text {elec }}+l \cdot \varepsilon_{\mathrm{fs}} \cdot d^{2} \text { if } d<d_{0}, \\
2 l \cdot E_{\mathrm{elec}}+l \cdot \varepsilon_{\mathrm{mp}} \cdot d^{4} \text { if } d \geq d_{0}
\end{array}\right.
$$

where:

$E_{\text {elec }}$ is the energy dissipation to run the radio,

$\varepsilon_{\mathrm{fs}} \quad$ is the free space model of transmitter amplifier,

$\varepsilon_{\mathrm{amp}}$ is the multi-path model of transmitter amplifier,

$l \quad$ is the data length,

$d_{0} \quad$ is the distance threshold.

The total energy consumed to transmit one bit data over $n$-hop is given as

$$
\begin{aligned}
E(n)=\left(E_{\text {elec }}+\varepsilon_{\text {amp }} \cdot d_{1}^{\alpha}\right) & +\sum_{i=2}^{n}\left(2 E_{\text {elec }}+\varepsilon_{\text {amp }} \cdot d_{i}^{\alpha}\right)=(2 n-1) E_{\text {elec }}+ \\
& +\sum_{i=1}^{n} \varepsilon_{\text {amp }} \cdot d_{i}^{\alpha},
\end{aligned}
$$

where $\varepsilon_{\mathrm{amp}}=\varepsilon_{\mathrm{fs}}$ when $\alpha=2$ and $\varepsilon_{\mathrm{amp}}=\varepsilon_{\mathrm{mp}}$ when $\alpha=4$.

The advantage of DEAR is that, it achieved better energy efficiency and energy balancing. [10] Proposes an Address light Integrated MAC and Routing Protocol (AIMRP) to increase the overall lifetime of a Wireless Sensor Network. The author has provided mathematical and simulation model for his work and has compared the result with SMAC. AIMRP [10] organizes network into concentric tiers around the sink, and routes event report by forwarding them from one tier to another, in the direction of the sink. If a node in a particular tier has to send a data packet to the sink, it will broadcast an RTR packet which includes RSD (Randomly chosen Source ID), STD (Source Tier ID), NAV (Network Allocation Vector) and OPI (Optional Packet Information). Any node in the previous tier can respond with a CTR for this RTR. The CTR includes RSD, STD, RRD (Randomly chosen Receiver ID), RTD (Receiver Tier ID) and NAV. Once the CTR is received, the source and receiver will quickly exchange a DATA and ACK packet. Like this, the data packet moves from one tier to another until it reaches the sink. The message format of AIMRP is shown in Fig. 5. AIMRP is address light in which it does not employ unique per node addressing (Fig. 6) and is integrated since the next hop is identified using the MAC control packets via an any cast query.

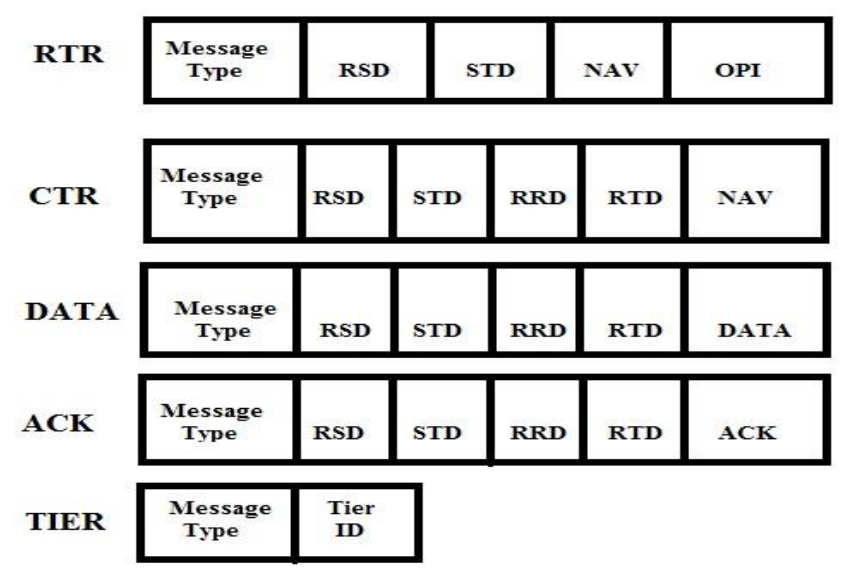

Fig. 5. Message format of AIMRP 


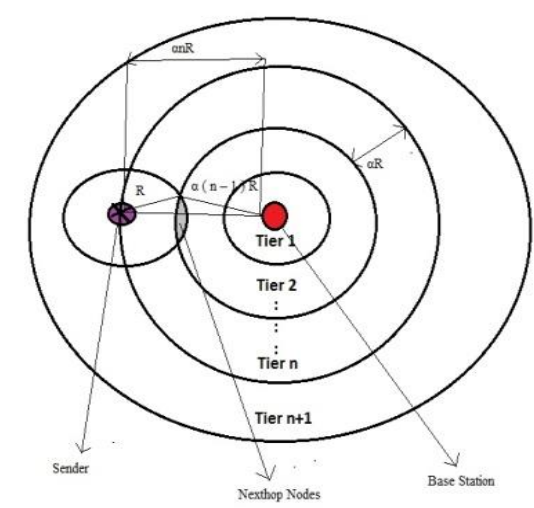

Fig. 6. An Address-Light, Integrated MAC and Routing protocol for WSN

Average power consumption in AIMRP is calculated as

$$
P_{\mathrm{avg}}=N\left(E_{\mathrm{up}}+E_{\mathrm{dw}}+P_{\mathrm{on}} t_{\mathrm{on}}\right) \sigma+\frac{E_{\text {report }}}{T},
$$

where:

$N \quad$ is the mean number of sensor nodes,

$E_{\text {up }}$ is the energy required to power the radio of a node ON,

$E_{\mathrm{dw}}$ is the energy required to power the radio of a node OFF,

$P_{\text {on }}$ is the power consumption with the radio on,

$t_{\text {on }}$ is the on-period in power saving mode,

$\sigma \quad$ in $\frac{1}{\sigma}$ is the mean sleeping interval,

$E_{\text {report }}$ is the energy consumed per event report.

The advantage of AIMRP [10] is that it outperforms S-MAC for event-detection application in terms of network lifetime. Its limitation is that it assumes, only one node detects the event and there is no provision to handle burst in the traffic.

[11] uses a mathematical model to analyze the performance gains by adding additional sensor nodes around sinks or base stations. It has also been proven analytically that by placing additional sensor nodes around the sink the hotspot problem can be solved. Adding a limited number of nodes can drastically increase the lifetime of certain networks.

The paper partitions the set of all sensor nodes $V$ into non-empty subsets $S_{0}, S_{1}, \ldots, S_{n}$, satisfying $V=S_{0} \cup S_{1} \cup \ldots \cup S_{n} ; S_{i}$ is the set of nodes reachable from base station $B$ in $i$ hops, but not less than $i$ hops. Hence, $S_{0}=\{B\}, S_{i}$ is the sphere of radius $i$ around $S_{0}, B_{i}$ is the ball of radius $i$, where $B_{i}=S_{0} \cup S_{1} \cup \ldots \cup S_{i}$,

$$
s_{i}=\left|S_{i}\right|, b_{i}=\left|B_{i}\right| \text {, and } N=|V| \text {. }
$$

Let $r$ be the energy consumed to receive one packet and let $t$ be the energy consumed to transmit one packet. Hence, we get

$$
m_{i}=\frac{N-b_{i}}{s_{i}} r+\frac{N-b_{i}+s_{i}}{s_{i}} t,
$$

$N-b_{i}$ denotes the total number of nodes outside $B_{i}$, i.e., the total number of packets that the set of nodes in sphere $S_{i}$ received in each iteration. The nodes in $S_{i}$ must forward $N-b_{i}+s_{i}$ packets in each iteration, i.e., the packets received from outer 
sphere plus their own $s_{i}$ sensor readings. The best a routing algorithm can do is to equally distribute the energy consumption for receiving and transmitting packets across all the nodes in $S_{i}$, therefore the denominator is $s_{i} ; m_{i}$ represents the energy consumption in a particular sphere.

Adding $k_{i}$ additional nodes to sphere $S_{i}$ the previous Equation (7) can be rewritten as

$$
m_{i}^{\prime}=\frac{N-b_{i}}{s_{i}+k_{i}} r+\frac{N-b_{i}+s_{i}}{s_{i}+k_{i}} t,
$$

$m_{i}^{\prime}<m_{i}$, hence adding more nodes around sink will reduce the impact of the hotspot. [14] Investigates the effect of multiple battery levels to maximize the useful lifetime of the network. In [15] is analyzied the power consumption characteristics of a WSN and presents a technique that performs aggressive energy optimization to enhance the network lifetime by orders of magnitude. In [16] is provided a novel approach for energy-aware and context-aware routing of sensed data. It uses a network clustering and assigns a less-energy-constrained gateway node that acts as a network manager. In [17] is proposed a design, which is used to create proper sized Hotspots integrated with a clustering mechanism, thereby maximizing the network lifetime. In [18] is proposed a hybrid approach that combines flat multi-hop routing and hierarchical multi-hop routing to mitigate the Hotspot problem. The paper analyses the power consumption in the hotspot area. Energy consumption corresponding to hotspot area is given as

$$
E^{\text {hotspot }}=\lambda \cdot E(d) \cdot M,
$$

where $\lambda$ is average number of hops the data has to be relayed through in hotspot to reach the sink $(r / d)$,

$E(d)$ is the energy consumed to transmit a unit of data over a distance $d,\left(\varepsilon, d^{2}\right)$, $M$ is the volume of data,

$\lambda$ is the $r / d$,

$r$ is the hotspot area.

Volume of data flowing into a hotspot, $M$ can be derived as

where: $m$ is the message size,

$$
M=m N\left(1-\frac{\pi r^{2}}{4 l^{2}}\right),
$$

$N$ is the number of nodes in the network,

$l$ is the length of the area;

$$
\begin{aligned}
E^{\text {hotspot }} & =\left(\frac{r}{d}\right) * \varepsilon_{1} d^{2}+m N\left(1-\frac{\pi r^{2}}{4 l^{2}}\right)= \\
& =\varepsilon_{1} \operatorname{mrd} N\left(1-\frac{\pi r^{2}}{4 l^{2}}\right), \\
E_{\text {flat }}^{\text {hotspot }} & =\varepsilon_{1} \operatorname{mrd}_{\text {flat }} N\left(1-\left(\frac{\pi r^{2}}{4 l^{2}}\right)\right), \\
E_{\text {hierarchical }}^{\text {hotspot }} & =\varepsilon_{1} \operatorname{mrd}_{\text {hierarchical }} \sigma N\left(1-\left(\frac{\pi r^{2}}{4 l^{2}}\right)\right), \\
E_{\text {hybrid }}^{\text {hotspot }} & =\varepsilon_{1} \operatorname{mrd}_{\text {flat }} \sigma N\left(1-\left(\frac{\pi r^{2}}{4 l^{2}}\right)\right),
\end{aligned}
$$


where $d_{\text {flat }}$ and $d_{\text {hierarchical }}$ are the average transmission distances for nodes when flat and hierarchical multi-hop routing algorithms are used respectively, and $\sigma$ is the compression ratio which is defined as

$$
\sigma=\frac{\text { Size[CompressedData] }}{\text { Size[OriginalData] }}
$$

i.e., the dividend gained by employing hybrid multi-hop routing will be more than the flat or hierarchical routing. In [19] is optimized the distance of each individual node, so that all sensor nodes consume their energy at a similar rate. In [20] is used Mobile Sensor Nodes for Surveillance. In [21] is discussed the problem of radio interface in WSN and proposed a method to solve it using reconfigurable IR-UWB radio. In [22] is proposed a Refined Integrated MAC and Routing Protocol to mitigate the impact of the hotspot.

$\mathrm{Sp}$ oorthi, S a h a and Mathur [23] proposed a mechanism to detect sensor node failures on the basis of the delay incurred in propagation and also the energy associated with sensors in the field of deployment. The algorithm plays in the best possible way to detect the failure in sensors. The Boolean sensing model is considered to calculate the network coverage of the wireless sensor network for various numbers of nodes in the network.

MaximuM-LEACH [24] provides a solution by load balancing the number of nodes equally by fixing the average value $N$, so the life time of the network is increased.

Sarasvathi and Iyengar [25] proposed a Multi Route Rank based Routing (MR3) protocol, which enhances the link dynamics for Industrial Wireless Mesh Sensor Networks (IWMSN) and also provides interference free reliable packet delivery in harsh environments. The rank of a node is estimated based on density, hop count, energy and Signal to Interference plus Noise Ratio (SINR). The route discovery phase finds the rank value to forward the data packet in a reliable path. Once the forwarding path is established, subsequently the data packets can be propagated towards the destination without using any location information.

Sink-based Centralized transmission scheduling (SC-Sched) [26] integrates asymmetric communication and wake-up radio. In SC-Sched [26] sensor nodes do not update routing information. Instead, a sink node collects node position information and on this basis determines a tree-based routing and a transmission order. Each sensor node is equipped with a wake-up receiver. At the time of transmission of a packet between two sensor nodes, the sink node transmits a Wakeup Call $(\mathrm{WuC})$ using a transmission power larger than the one used for data transmission, directly activating both transmitting and receiving sensor nodes simultaneously to start the communication.

In [27] is proposed a quantification algorithm to reduce the impact of the hotspot problem in WSN. It uses sensor nodes with similar initial energy levels. In the present work we are using a protocol called RIMRP [22] (Refined Integrated MAC and Routing Protocol) along with a novel quantification algorithm, custom designed for sensor nodes with varying Initial energy levels. The present work uses sensor nodes with varying initial energy levels at different tiers. The present work outperforms $[22,27]$ in terms of improved Network lifetime and overall energy consumption. 


\section{Proposed work}

The proposed work is divided into three parts viz., 3.1 Tier formations with refined Integrated MAC and Routing Protocol, 3.2 Quantification algorithms for Sensor Nodes with varying initial energy levels and 3.3 Mathematical model for Energy Consumption.

\subsection{Tier formation with refined integrated MAC and Routing protocol}

To create tiers, we are following the approach similar to $[10,27]$ with additional refinement as proposed in [22]. We assume that the nodes are operating at a frequency of $2.4 \mathrm{GHz}$ with a transmission range of $R$ meters. We divide the nodes around the sink as tiers. The nodes closer to the sink (e.g., within a range of $R$ meters) form Tier 1 . The next level of nodes (e.g., which have a range of distance between $R$ meters and $2 R$ meters) form Tier 2 . It follows the same calculation of the range of distance for the rest of the tiers. Tier formation consists of two phases namely, 1) Setup Phase, and 2) Action Phase.

Setup Phase: Initially all nodes except the sink node's tier id will be set as -1 and the tier id of the sink will be 0 . The sink will broadcast a Hello Message with its tier id (i.e., 0) with a power level corresponding to the communication range of $\alpha \mathrm{R}$ where $R$ is the transmission range of the node and $\alpha=0.45$ as suggested in AIMRP [10]. This ensures that the nodes near the tier boundaries can also transmit messages to the next tier. The nodes receiving this message will set their tier id as 1 (i.e., received Hello Message tier id +1 ). Now all these nodes with tier id 1 will broadcast the Hello Message. The nodes with tier id -1 receiving this hello message will update their tier id to 2 . This process continues until all nodes are assigned with a positive tier id. Thus, the tier-formation will be completed at the end of the setup phase.

Action Phase: If any node has to send a sensed data, the node will broadcast an RTS message with its tier id. Unlike [10] All nodes in the previous tier receiving this RTS message (i.e., the tier id of the receiving node should be 2 to send a CTS if the sender's tier id is 3) can respond with a CTS message. In contrary to [10] along with the CTS message, the node will also send its current energy level [22]. Upon receiving the CTS message from all the nodes the sender will unicast DATA packet to the node whose energy level is maximum [22]. The receiver node would send an acknowledgment to the sender. Now, the receiver node will become the new sender and it will broadcast the RTS and transmit the data packet to the next tier. This process continues until the DATA packet successfully reaches the sink.

The intrinsic nature of communication in a WSN is from the node to sink and not from node to node.

3.2. Quantification Algorithm for Sensor nodes with varying initial (battery) energy levels

The quantification algorithm for sensor nodes with varying initial energy levels is discussed with the following example. 
In Fig. 7, there are three nodes A, B and C in Tiers 1, 2 and 3, respectively. Let us assume that all the three nodes have an initial energy level of $5 \mathrm{~nJ}$ and assume that for every communication the node loses $1 \mathrm{~nJ}$ of energy. Say, for a given instance of time, all three nodes would like to communicate a sensed parameter to the sink node.

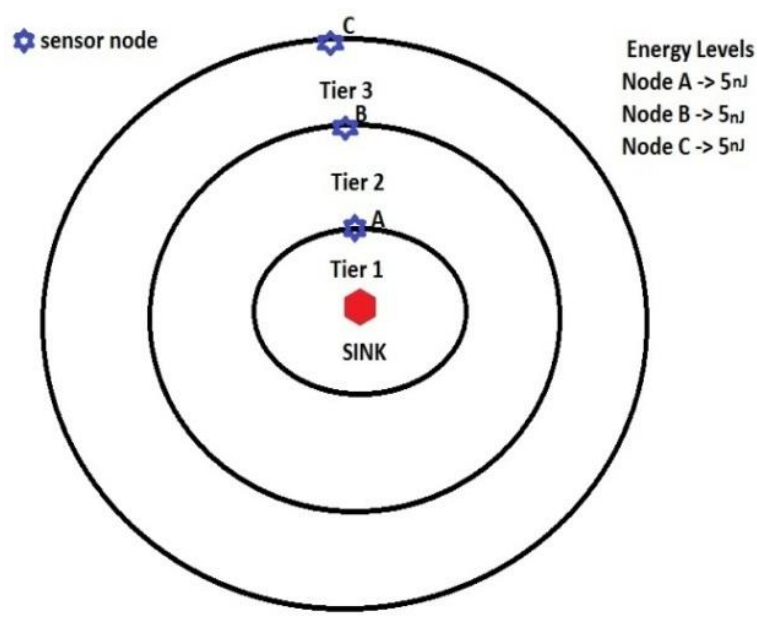

Fig. 7. Hotspot problem

Node $\mathrm{C}$ will communicate the sensed data to node $\mathrm{B}$ in concurrence with the tier-based multi-hop communication. Now node C's available energy will be $4 \mathrm{~nJ}$. Node B has to communicate its own data besides the data packet received from C, so its energy will become $3 \mathrm{~nJ}$ and node A has to communicate its own data besides communicating two data packets from B. So its energy will be $2 \mathrm{~nJ}$. At the end of cycle 1, node A's energy will be $2 \mathrm{~nJ}$, node B's energy will be $3 \mathrm{~nJ}$ and node C's energy will be $4 \mathrm{~nJ}$. Consider the same action for Cycle 2, i.e., at a particular time instance, all three nodes would like to communicate the sensed parameter to the sink. Now, after C's communication, its energy will be $3 \mathrm{~nJ}$ and after B communicating its own sensed data and data packet from C, its energy will be $1 \mathrm{~nJ}$. A's available energy is $2 \mathrm{~nJ}$, but it has to transmit 3 data packets, whereas it can communicate only 2 data packets and after that node A will be drained and as a result Hotspot occurs and the network gets isolated.

This research work proposes a simple quantification algorithm for sensor nodes having varying energy (battery) levels. After analyzing the above-stated problem, the quantification algorithm is defined as follows.

If there are $n$ tiers and $x$ nodes in each tier, then the energy (battery) level of any node in a given tier should be $e_{i}$ (where $i$ represent the tier number), given the energy level $e_{n}$ of every individual sensor node in $n$-th tier, the total energy $(E)$ of the network is given as

$$
E=x e_{1}+x e_{2}+x e_{3}+\cdots+x e_{n},
$$

where $e_{i}=[n-(i-1)] e_{n}, 1 \leq i \leq n-1$,

$$
e_{n-1}<e_{n-2}<\cdots<e_{2}<e_{1},
$$


So, $e_{1}$, i.e., the nodes in Tier 1 will have the maximum initial energy level, and the nodes in tier $n$ will have the minimum energy level. The energy distribution of nodes in various tiers is given in Table 1.

Table 1. Energy distribution based on Quantification algorithm

\begin{tabular}{|c|c|l|c|}
\hline No & Energy level & Description & Value \\
\hline 1 & $e_{n}$ & Energy of every node in Tier $n$ & Constant (fixed/base value) $e_{n}$ \\
\hline 2 & $e_{n-1}$ & Energy of every node in Tier $n-1$ & $2 e_{n}$ \\
\hline 3 & $e_{n-2}$ & Energy of every node in Tier $n-2$ & $3 e_{n}$ \\
\hline 4 & $e_{1}$ & Energy of every node in Tier 1 & $n e_{n}$ ( $n$ is the number of Tiers) \\
\hline
\end{tabular}

By applying quantification algorithm with varying energy levels to Fig. 7, we will get a setup as shown in Fig. 8 .

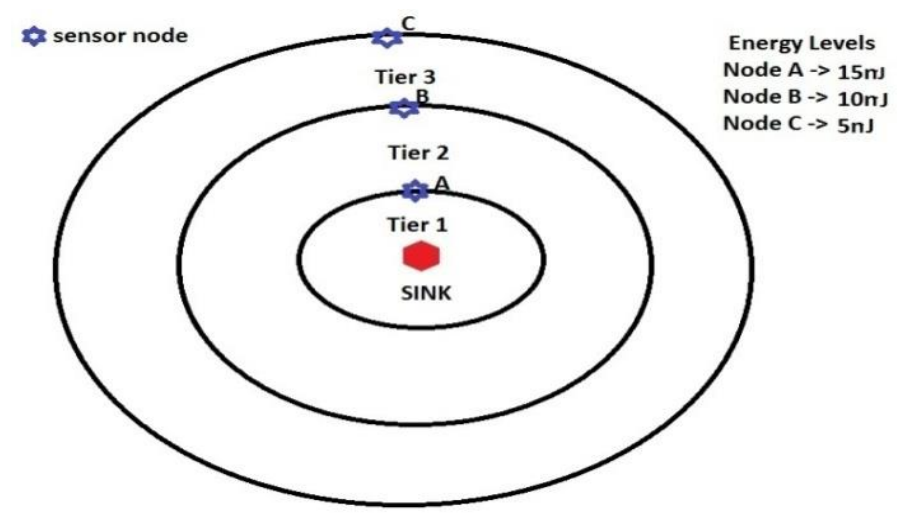

Fig. 8. Nodes having Varying Energy Levels based on Quantification algorithm

Now, consider the situation that at a given instance of time, one node from each tier would like to communicate with the sink and also let us assume that applying quantification algorithm the initial energy of node $\mathrm{C}$ is $5 \mathrm{~nJ}$, node $\mathrm{B}$ is $10 \mathrm{~nJ}$ and node A is $15 \mathrm{~nJ}$. Now during Cycle 1 node $\mathrm{C}$ is sending a sensed data to Tier 2 (node B) and C's energy will become $4 \mathrm{~nJ}$. And node B will forward its own data and data from C to Tier 1 (node A), so the energy of node B will be $8 \mathrm{~nJ}$. Similarly, node, A will forward two data packets from B and its own data packet, so the energy of a node A will be $12 \mathrm{~nJ}$. So, by the end of cycle 1 node, A's energy level will be $12 \mathrm{~nJ}$, the node B's energy level will be $8 \mathrm{~nJ}$ and node C energy level will be $4 \mathrm{~nJ}$. And if this continues at the end of Cycle 5 all nodes energy will be $0 \mathrm{~nJ}$. It should be noted here that the events occur rarely in a WSN. What we have considered here is a worst-case scenario to produce a model for maximizing the lifetime of WSN. Compared to the basic model proposed in Fig. 7, the model proposed in Fig. 8 increases the lifetime by 4 -fold, which is a substantial improvement.

3.3. Mathematical representation for energy consumption and network lifetimefor WSN having sensor nodes with varying initial energy levels

The notation used for these calculations is defined in Table 2. 
Table 2. Notations

\begin{tabular}{|c|c|l|}
\hline No & Notation & \multicolumn{1}{c|}{ Description } \\
\hline 1 & $N$ & Total number of nodes in the Sensor Network \\
\hline 2 & $n$ & Number of Tiers \\
\hline 3 & $x$ & Number of nodes in each tier \\
\hline 4 & $T_{x}$ & The Energy required to transmit one packet \\
\hline 5 & $R_{x}$ & The Energy required to receive one packet \\
\hline 6 & $i$ & Current tier number $(1 \leq i \leq n)$ \\
\hline 7 & $E_{i}$ & Energy Consumed at Tier $i$ for one Round \\
\hline 8 & $\varepsilon$ & The Energy spent in control packets \\
\hline 9 & NLT & Network Lifetime \\
\hline
\end{tabular}

Energy consumed at Tier $i$ for one Round is given as

$$
E_{i}=\frac{\left\{[N-i x] R_{x}\right\}}{x}+\frac{\left\{[N-(i-1) x] T_{x}\right\}}{x}+\frac{\varepsilon}{x},
$$

$[N-i x]$ represents the sum of nodes in Tier $i+1$, Tier $i+2$, Tier $i+3, \ldots$, Tier $n$. Let us assume $N=25, n=5$ and $x=5$ and $i=3$ then $[N-i x]=25-3(5)=10$, i.e., Tier 3 should receive data packets from 10 nodes. These 10 nodes are spread across Tier 4 and Tier 5;

$[N-(i-1) x]$ represents the sum of nodes in Tier $i$, Tier $i+1$, Tier $i+2, \ldots$, Tier $n$. With the above assumptions. $[N-(i-1) x]=25-2 \times 5=15$, i.e., Tier 3 should transmit the data packets of 15 nodes. These 15 nodes are spread across Tier 3 , Tier 4 and Tier 5.

Each part of Equation (17) is divided by $x$ to represent that the load is uniformly distributed to all nodes in the particular tier.

In most cases $\max \left\{E_{\mathrm{I}}\right\}$ will be $E_{1}$.

\subsection{Results and discussion}

This research work aims to evaluate the performance of RIMRP for WSN having sensor nodes with varying initial energy levels in terms of network lifetime. The present work is simulated in NS2.35 with 12 scenarios, each having three tiers. All scenarios have 4 nodes in each tier as shown in Fig. 9. The nodes with varying initial energy (battery) levels are distributed in each tier as depicted in Table 3.

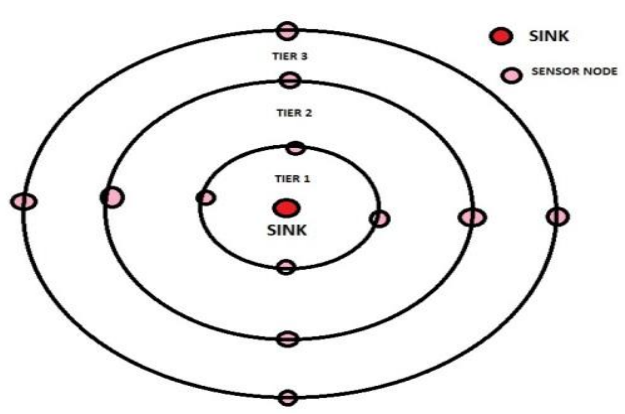

Fig. 9. Simulation setup 
Table 3. Scenarios

\begin{tabular}{|c|c|c|c|}
\hline \multirow{2}{*}{$\begin{array}{c}\text { Scenario } \\
\text { No }\end{array}$} & \multicolumn{3}{|c|}{ Energy levels, nJ } \\
\cline { 2 - 4 } & Tier 1 & Tier 2 & Tier 3 \\
\hline 1 & 10 & 10 & 10 \\
\hline 2 & 30 & 20 & 10 \\
\hline 3 & 30 & 20 & 11 \\
\hline 4 & 30 & 20 & $12 / 13$ \\
\hline 5 & 30 & 20 & 14 \\
\hline 6 & 30 & 20 & 15 \\
\hline 7 & 30 & 20 & 16 \\
\hline 8 & 30 & 20 & 17 \\
\hline 9 & 30 & 20 & $18 / 19 / 20$ \\
\hline 10 & 30 & 21 & 19 \\
\hline 11 & 30 & 21 & 20 \\
\hline 12 & 30 & 40 & 20 \\
\hline
\end{tabular}

It can be observed from the above table that row 2 specifies the energy distribution as of the quantification algorithm to maximize the network lifetime, i.e., all nodes in Tier 1 have energy levels of $30 \mathrm{~nJ}$, all nodes in Tier 2 have energy levels of $20 \mathrm{~nJ}$ and all nodes in Tier 3 have energy levels of $10 \mathrm{~nJ}$.

These 12 scenarios have been simulated with S-MAC and Refined Integrated MAC and Routing protocol (RIMRP). Each scenario is executed for 10 times. In each round of execution, a random node is selected to communicate with sink. The values plotted in the graph are the average values of the simulation results. From the simulation results it has been observed that the quantification algorithm performs better with RIMRP than with S-MAC. The quantification algorithm gives a direction about distribution of energy level among various tiers. From row numbers 3-12 in Table 4 and Table 5 we can observe a reasonably good increase in the network life time with small increase of energy levels in Tier 3 and Tier 2. Basically, the quantification algorithm gives a direction to distribute energy among various tiers; one need not follow it strictly. Table 4 and Table 5 depict the average network lifetime of RIMRP and S-MAC, respectively.

Table 4. RIMRP

Table 4. RIMRP
\begin{tabular}{|c|c|c|c|c|}
\hline \multirow{2}{*}{ Scenario No } & \multicolumn{3}{|c|}{ Energy levels, $\mathrm{nJ}$} & \multirow{2}{*}{ Average N/w life time, s } \\
\cline { 2 - 4 } & Tier 1 & Tier 2 & Tier 3 & 91.9 \\
\hline 1 & 10 & 10 & 10 & 107.56 \\
\hline 2 & 30 & 20 & 10 & 120.52 \\
\hline 3 & 30 & 20 & 11 & 170 \\
\hline 4 & 30 & 20 & $12 / 13$ & 172 \\
\hline 5 & 30 & 20 & 14 & 183 \\
\hline 6 & 30 & 20 & 15 & 195 \\
\hline 7 & 30 & 20 & 16 & 207 \\
\hline 8 & 30 & 20 & 17 & 232.28 \\
\hline 9 & 30 & 20 & $18 / 19 / 20$ & 249.04 \\
\hline 10 & 30 & 21 & 19 & 249.12 \\
\hline 11 & 30 & 21 & 20 & 261.04 \\
\hline 12 & 30 & 40 & 20 & \\
\hline
\end{tabular}




Table 5. S-MAC
\begin{tabular}{|c|c|c|c|c|}
\hline \multirow{2}{*}{ Scenario No } & \multicolumn{2}{|c|}{ Energy levels, $\mathrm{nJ}$} & \multirow{2}{*}{ Avgerage N/w life time, $\mathrm{s}$} \\
\cline { 2 - 4 } & Tier 1 & Tier 2 & Tier 3 & \\
\hline 1 & 10 & 10 & 10 & 79.5 \\
\hline 2 & 30 & 20 & 10 & 90.4 \\
\hline 3 & 30 & 20 & 11 & 102.42 \\
\hline 4 & 30 & 20 & $12 / 13$ & 132.6 \\
\hline 5 & 30 & 20 & 14 & 134.5 \\
\hline 6 & 30 & 20 & 15 & 143.2 \\
\hline 7 & 30 & 20 & 16 & 151.5 \\
\hline 8 & 30 & 20 & 17 & 160.5 \\
\hline 9 & 30 & 20 & $18 / 19 / 20$ & 181.7 \\
\hline 10 & 30 & 21 & 19 & 191.12 \\
\hline 11 & 30 & 21 & 20 & 192.3 \\
\hline 12 & 30 & 40 & 20 & 205.5 \\
\hline
\end{tabular}

The Scenario 2 of Table 4 represents energy distribution as of the quantification algorithm and it provides an average network lifetime of $107.56 \mathrm{~s}$. The average network lifetime increases by adding more nodes in Tier 3, which can be observed in Table 4 from Scenario 3 to Scenario 9. However, after that there is no notable improvement in the network lifetime by adding more nodes in Tier 3. But, when we increase the number of nodes in Tier 2, there is a significant improvement in the network lifetime. Hence, the quantification algorithm can be used to identify the distribution of energy in an optimal way. Fig. 10 and Fig. 11 show the performance analysis of Quantification algorithm with sensor nodes having varying energy (battery) levels for RIMRP and S-MAC. From the Fig. 10 and Fig. 11, it can be observed that RIMRP outperforms S-MAC in terms of average network lifetime.

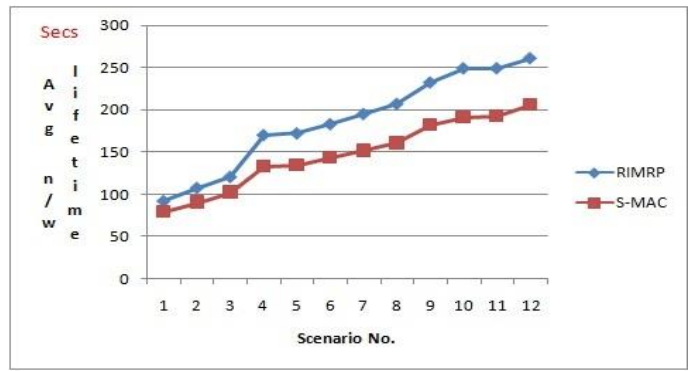

Fig. 10. RIMRP and S-MAC using sensor nodes with varying energy levels - Line Graph

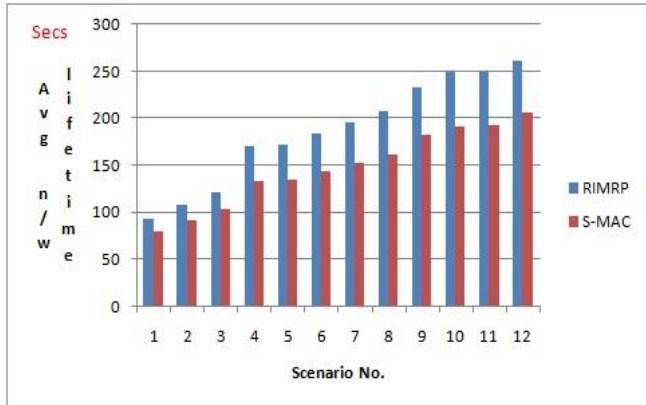

Fig. 11. RIMRP and S-MAC using sensor nodes with Varying Energy Levels-Bar Graph 
Figs 12 and 13 show the performance analysis of RIMRP and S-MAC without using quantification algorithm and using sensor nodes with similar energy levels of $8 \mathrm{~nJ}$ each. For each execution, 45 nodes are deployed randomly and their network lifetime is calculated for RIMRP and S-MAC. The total energy available in the network is $360 \mathrm{~nJ}$ ( $8 \mathrm{~nJ} \times 45$ nodes) which is equal to the Scenario 12 of Table 4 and Table $5(30 \mathrm{~nJ} \times 4$ nodes $+40 \mathrm{~nJ} \times 4$ nodes $+20 \mathrm{~nJ} \times 4$ nodes $=360 \mathrm{~nJ})$. From Figs 12 and 13 , we can observe that the average network lifetime of RIMRP for 8 executions is $164.25 \mathrm{~s}$ and the average network lifetime of S-MAC for 8 iterations is 154.0. Whereas the average network lifetime of RIMRP with quantification algorithm (Scenario 12, Table 4) is 261.04 and the average network lifetime of S-MAC with quantification algorithm (Scenario 12, Table 5) is 205.5. This shows the role of the quantification algorithm in improving the network lifetime. Also, we can observe that RIMRP outperforms S-MAC in terms of network lifetime.

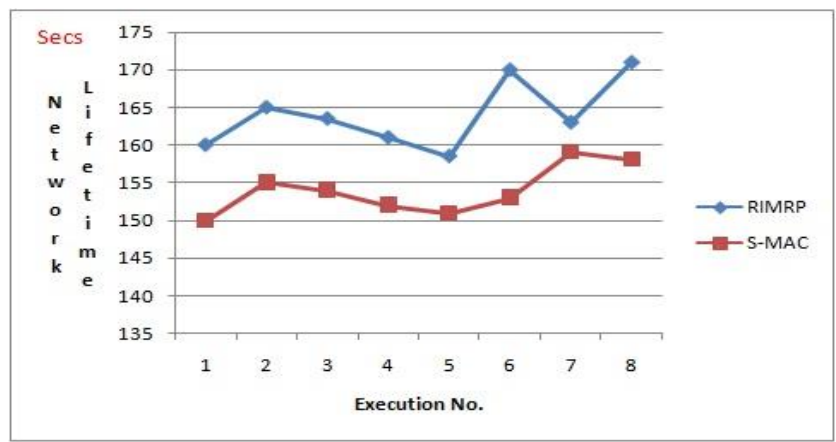

Fig. 12. S-MAC and RIMRP without Using Quantification Algorithm and Using Sensor Nodes Having Similar Energy Levels - Line Graph

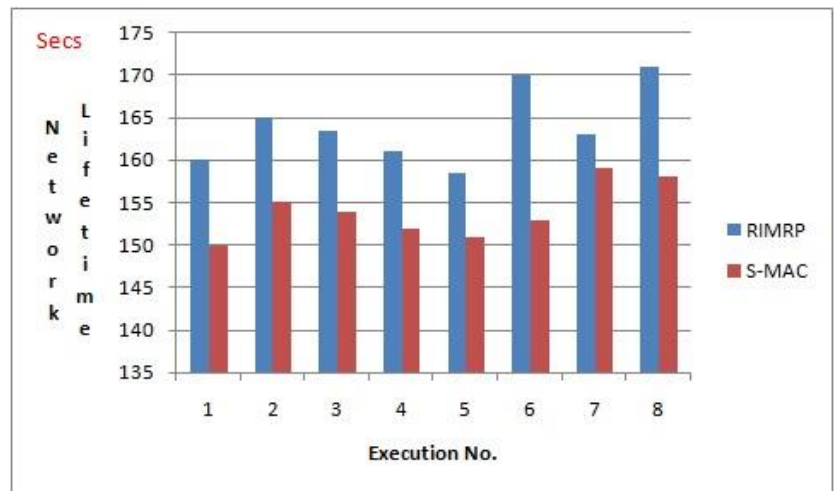

Fig. 13. S-MAC and RIMRP without Using Quantification Algorithm and Using Sensor Nodes Having Similar Energy Levels - Bar Graph

Figs 12 and 13 show the importance of quantification algorithm. The simulation setup for above figures are similar to the Scenario 12 of Table 4 with respect to overall energy levels, but there is a vast difference in the network lifetime. This shows the impact of using sensor nodes with varying energy levels and distributing it across the tiers based on quantification algorithm. 


\section{Conclusion and open issues}

In this paper, we have provided a simple solution to reduce the impact of Hotspot for sensor nodes with varying initial energy levels by proposing a quantification algorithm and by using the Refined Integrated MAC and Routing Protocol. The simulated result shows that the proposed work outperforms SMAC in terms of network lifetime. The present simulation assumes a 2D WSN, but most of the real networks are 3D. Hence as an extension of this work a 3D WSN can be considered. This simulated work can also be tried with a real test-bed.

\section{References}

1. Akyildiz, I. F, M. C. Vuran. Wireless Sensor Networks. A John Wiley and Sons, Ltd., Publication, United Kingdom, 2010, pp. 1-15.

2. Akyildiz, I., F. Su, W. Sankarasubramania m, Y. Cayirci. Wireless Sensor Networks: A Survey. - Computer Networks, 2002, pp. 393-422.

3. S h a r m a, S., R. K. B a n s a l, S. B a n s a l. Issues and Challenges in Wireless Sensor Networks.IEEE Conference on Machine Intelligence Research and Advancement, December 2013, pp. 1-5.

4. He in zelman, W. R., A. Chandrakas an, H. B a lakrishnan. Energy-Efficient Communication Protocol for Wireless Microsensor Networks. - In: Proc. of IEEE Hawaii International Conference on System Sciences, January 2000, pp. 1-10.

5. Y o u n i s, O., S. F a h m y. HEED: A Hybrid, Energy-Efficient, Distributed Clustering Approach for ad hoc Sensor Networks. - IEEE Transactions on Mobile Computing, Vol. 3, 2004, No 4, pp. 366-379.

6. Lind sey, S., C. S. Raghavendra. PEGASIS: Power-Efficient Gathering in Sensor Information Systems. - IEEE Aerospace Conference Proceedings, March 2002, pp. 1125-1130.

7. W a n g, Y., T. L. X. Y a n g, D. Z h a n g. An Energy Efficient and Balance Hierarchical Unequal Clustering Algorithm for Large Scale Sensor Networks. - Information Technology Journal, Vol. 8, 2009, No 1, pp. 28-38.

8. W e i., D., Y. J i n, S. V u r a 1, K. Mo e s s n e r, R. T a f a z o 11 i. An Energy-Efficient Clustering Solution for Wireless Sensor Networks. - IEEE Transactions on Wireless Communications, Vol. 10, December 2011, No 11, pp. 3973-3983.

9. W a n g, J., J. K i m, L. S h u, Y. N i u, S. L e e. A Distance-Based Energy Aware Routing Algorithm for Wireless Sensor Networks. - Sensors, Vol. 10, 2010, No 10, pp. 9493-9511.

10. Kulkarni, S., A. Iy e r., C. R o s e n ber g. An Address-Light Integrated MAC and Routing Protocol for Wireless Sensor Networks. - IEEE/ACM Transactions on Networking, Vol. 14, 2006, No 4, pp. 793-806.

11. Rivas, H., T. Voigt, A. D unkels. A Simple and Efficient Method to Mitigate the Hot Spot Problem in Wireless Sensor Networks. - IEEE GLOBECOM Wireless ad hoc and Sensor Networks, 2004, pp. 1-8.

12. S i c h it i u, M. L., R. D u t t a. Networking Technologies, Services, and Protocols; Performance of Computer and Communication Networks; Mobile and Wireless Communications Systems, Berlin, Heidelberg, Springer-Verlag, 2002, pp. 376-386.

13. Nazi r, B., H. Hasbullah. Mobile Sink Based Routing Protocol (MSRP) for Prolonging Network Lifetime in Clustered Wireless Sensor Network. - In: IEEE International Conference on Computer Applications and Industrial Electronics, 2010, pp. 624-629.

14. S i chiti u, M. L., R. Dutta. Benefits of Multiple Battery Levels for the Lifetime of Large Wireless Sensor Networks, NETWORKING'2005. Networking Technologies, Services, and Protocols; Performance of Computer and Communication Networks; Mobile and Wireless Communications Systems. - Lecture Notes in Computer Science, Vol. 3462, 2005, pp. $1440-1444$. 
15. Raghunathan, V., C. Schurgers, M. B. Srivastava. Energy-Aware Wireless Microsensor Networks. - Signal Processing Magazine, IEEE, Vol. 19, 2002, Issue 2, pp. 40-50.

16. Y oun is, M., M. Yous s ef, K. A r is ha. Energy-Aware Routing in Cluster-Based Sensor Networks. - Modeling, Analysis and Simulation of Computer and Telecommunications Systems. - MASCOTS, 2002, pp. 129-136.

17. Ye, M., E. Cha n, Guih a i. On Mitigating Hot Spots for Clustering Mechanisms in Wireless Sensor Networks. National Laboratory of Novel Software Technology, Nanjing University, China, Department of Computer Science, City University of Hong Kong, China, pp. 558-561. 1-4244-0507-6/06/\$20.00 @2006 IEEE.

18. A h med, E. A., A. A b du 11 a, H. N is hi y a ma, N. K a to. Graduate School of Information Sciences, Tohoku University. Extending the Lifetime of Wireless Sensor Networks : A Hybrid Routing Algorithm. - Computer Communications, Vol. 35, May 2012, Issue 9, pp. 1056-1063.

19. W a ng, J., I. J. D e D i e u, A. d e L e o n, D. J o s e, S. L e e, Y.-K. L e e, K. H e e. Prolonging the Lifetime of Wireless Sensor Networks via Hotspot Analysis, pp. 383-386. 978-0-7695-4107-5/10 \$26.00 ( 2010 IEEE DOI 10.1109/SAINT.2010.31.

20. T s e n g, Y.-C., Y.-C. W an g, K.-Y. Ch eng, Y.-Y. H s i e h. iMouse: An Integrated Mobile Surveillanceand Wireless Sensor System. Published by the IEEE Computer Society, National Chiao Tung University, 2007, pp. 60-66.

21. Le c o in tre, A., D. D r a g o m i r e s c u, R. P l a n a. Design and Hardware Implementation of a Reconfigurable Mostly Digital IR-UWB Radio. - Romanian Journal of Information Science and Technology, Vol. 11, 2008, No 4, pp. 295-318.

22. B a l a murali, R., K. Kathiravan. A Refined Integrated MAC and Routing Protocol with Quantification Algorithm to Mitigate Hotspot Problems in Wireless Sensor Networks. Journal of Science and Technology, Vol. 8, August 2015, No 17, pp. 1-7. DOI:10.17485/ijst/2015/v8i17/68660.

23. S p o o r th i, K., S. S a h a, A. M a th u r. Discrete Path Selection and Entropy Based Sensor Node Failure Detection in Wireless Networks. - Cybernetics and Information Technologies, Vol. 16, 2016, No 3, pp. 137-153.

24. $\mathrm{K} \mathrm{u} \mathrm{m}$ a r, S. A., P. I 1 a $\mathrm{g}$ o. A Modified LEACH Protocol for Increasing Lifetime of the Wireless Sensor Network. - Cybernetics and Information Technologies, Vol. 16, 2016, No 3, pp. 154-164.

25. S a r a s vathi, V., N. C. S. N. I ye ngar. A Multi Route Rank Based Routing Protocol for Industrial Wireless Mesh Networks. - Cybernetics and Information Technologies Vol. 16, 2016, No 4, pp. 73-86.

26. I w a t a, M., S. T a n g, S. O b a b a. Energy-Efficient Data Collection Method for Sensor Networks by Integrating Asymmetric Communication and Wake-Up Radio. - Sensors, Vol. 18, April 2018, No 4, pp. 1-17.

27. B a la murali, R., K. Kathiravan. Mitigating Hot Spot Problems in Wireless Sensor Networks Using Tier-Based Quantification Algorithm. - Cybernetics and Information Technologies, Vol. 16, 2016, No 1, pp. 73-79.

Received: 19.12.2018; Second Version:30.05.2019; Accepted: 24.07.2019 